\title{
A MODIFIED FCM WITH OPTIMAL PEANO SCANS FOR IMAGE SEGMENTATION
}

\author{
A. Hafiane, B. Zavidovique \\ Institut d'Electronique Fondamentale \\ University of Paris-sud \\ 91405 Orsay, France
}

\author{
S. Chaudhuri \\ Department of Electrical Engineering \\ Indian Institute of Technology \\ Mumbai 400076, India
}

\begin{abstract}
This paper describes a new method for fuzzy segmentation based on spatial constraints. Taking into account the neighborhood influence two techniques are used. First a new feature is derived from Peano scans to represent a spatial relationship among neighbors. Second we incorporate a regularization term to Fuzzy C-means algorithm (FCM). The algorithm is tested on both synthetic and multispectral images. Experimental results are presented and discussed. They show the effectiveness of the method.
\end{abstract}

\section{INTRODUCTION}

Image segmentation is an essential task for image understanding and analysis. A large variety of methods have been proposed in the literature. Image segmentation can be defined as a classification problem where each pixel is assigned to a specific class. Clustering methods are considered as an unsupervised classification, where there is no need for prior knowledge about the data set. Many different clustering techniques have been proposed [1] [2] in computer vision and pattern recognition. Among those techniques Fuzzy c-means algorithm [3] which is widely used for image segmentation [4]. FCM algorithm allows points to belong to two or more classes with appropriate membership. This flexibility during the classification process is a useful property since it informs about the confidence attributed to a particular class or cluster. Although the original FCM algorithm yields good results for segmenting noisefree images, it fails to segment images corrupted by noise, outliers and other imaging artifact. Therefore segmenting real world images such as remote sensing images by FCM can lead to a erroneous segmentation. One way to deal with this problem is to filter image before applying FCM. However filtering may lose important information. It is advantageous to explicitly incorporate the neighborhood information along with pixel intensities in the image plane. Earlier works [5] show the improvement in segmentation results by incorporating the spatial information into the FCM algorithm. Pahm et al [6] propose a method by incorporat- ing a second constraint term to allow the membership function to be influenced by neighborhood membership functions. In the same context, Ahmed et al [7] add a second term to the FCM objective function in order to influence the classification of a given point by its neighborhood values. Those methods are based on direct contribution of neighbors' intensity values and do not take into account any other property of that neighborhood. Another approach which merges naturally the spatial context is Markov Random Field (MRF) [8]. It is widely used for segmentation problem, but computationally very expensive due to the complexity of parameters estimation. Generally segmentation methods are sensitive to contrast change or illumination conditions. We need to encode the neighborhood context in such a way as being:

1) Invariant to a shift in the pixel intensities as well as contrast changes

2) Able to encode a small number of parameters properties in a non-parametrized way as the estimation of such is often a very difficult task.

3) The encodeship should be local just as the pixel values so that the segmentation can be performed in a single pass.

Peano space filling curves have good topological properties [9] they preserve a spatial proximity, generally used to map a multi-dimensional space onto one dimension. Peano scans have been also used for mulitresolution image analysis [10]. However earlier works of [11] uses optimal Peano scans to generate a local patterns called motifs. It has been shown that method is efficient for texture analysis and image retrieval.

In this paper we present an approach based on spatial constraints for FCM. Two techniques are combined to incorporate the spatial context. The first one is an additional feature: rather than pixel intensity we introduce a new representation of neighborhood relationship by using local space filling curves. The second consists in modifying FCM objective function by adding a second term. This term allows the pixel to be influenced by its neighbors which yield a regularization effect. The proposed algorithm improves robustness to noise, outliers and retain simplicity. 
This paper is organized as follows. Section 2 describes the use of the local Peano scan to evaluate the relationship between adjacent pixels. Then, in the following section we present a new objective function for FCM. Our segmentation method is tested on both synthetic and real images, results are illustrated in section 4 . The paper is concluded in section 5 .

\section{FEATURE SELECTION}

\subsection{Optimal Scan}

The relationship among adjacent pixels is an important feature. It allows a better efficiency for image segmentation. In this section we describe a method that uses Peano space filling curves properties to encode the relationship in local neighborhood. Our method consists in determining the dependence between four adjacent pixels in term of intensity variation. A $2 \times 2$ pixels grid is traversed by a set of Peano curves to obtain a path which minimizes the intensity value over the scan, yielding an optimal scan. Given four adjacent pixels $\begin{array}{ll}p_{1} & p_{2} \\ p_{3} & p_{4}\end{array}$ the optimal scan follows the permutation $r^{*}$ that corresponds to

$$
\delta=\min _{r}\left\{\left|p_{r 1}-p_{r 2}\right|+\left|p_{r 2}-p_{r 3}\right|+\left|p_{r 3}-p_{r 4}\right|\right\}
$$

Fixing the start point in $2 \times 2$ grid yields a set of 6 possible paths (motifs). Changing the start point in the grid results in a different set of motifs. Using all possible scans results in 24 possible motifs. Referring to the minimization criterion

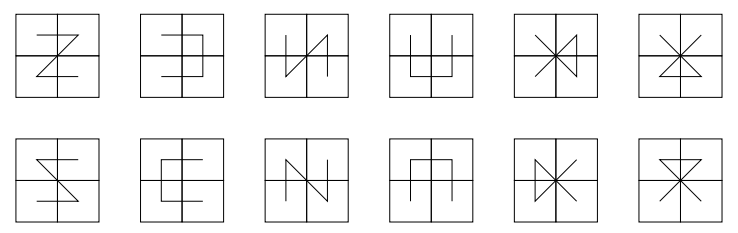

Fig. 1. 12 possible scans to account for all pairs traversing the four adjacent pixels

in Eq. 1, the number of motifs can be reduced to 12 as illustrated in Fig. 1. Note that given four adjacent pixels one of 12 paths yields a minimum value $\delta$. The advantage for using the optimal scan is that it brings information about adjacent pixels by minimizing the variation of intensity. Small value of $\delta$ means that there are less variation of intensity. In particular case where $2 \times 2$ pixels have the same values, all motifs scan optimally those pixels and $\delta$ is equal to 0 .

\subsection{Neighborhood Contribution}

Consider a set of grey level values of 8-connected pixels. The centre pixel and its neighbors are divided into four groups $g_{1}=\{o, a, b, c\}, g_{2}=\{o, c, d, e\}, g_{3}=\{o, e, f, g\}, g_{4}=$ $\{o, g, h, a\}$, where each group contains four adjacent points as illustrated in Figure 2. Using this configuration one can obtain $\delta$ (optimal scan) in each set. Then the four optimal values will be considered as a feature that represents the relationship between a given pixel and its neighbors. However in order to control the influence of this feature a weight $w$ is attributed. Finally the feature vector of a given pixel shows the flowing structure:

$$
x=\left\{a^{1}, \ldots a^{p}, w\left(\delta_{1}^{1}, \delta_{2}^{1}, \delta_{3}^{1}, \delta_{4}^{1}, \ldots, \delta_{1}^{p}, \delta_{2}^{p}, \delta_{3}^{p}, \delta_{4}^{p}\right)\right\}
$$

where $a^{p}$ represent intensity value in color plane $p$ and $\delta_{k}^{p}$ is the optimal scan of the group $g_{k}(k \in\{1,2,3,4\})$ in the $p$ th color plane.An advantage of using such feature is its invariance under uniform additive light or a sensor gain. Adding the same intensity to a four adjacent pixels do not affect the optimal path.

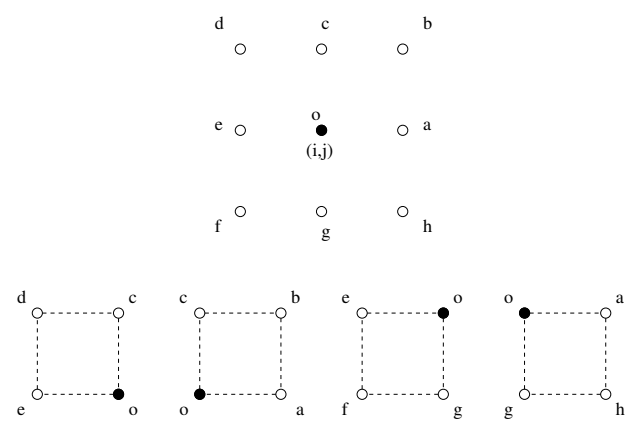

Fig. 2. Decomposition of $3 \times 3$ neighborhood in a set of four groups

\section{MODIFIED FCM}

The traditional FCM algorithm minimizes the objective function $J(U, V)$ which is defined by sum of similarity measures. The objective function is given by

$$
J(U, V)=\sum_{i=1}^{C} \sum_{j=1}^{N} u_{i j}^{m}\left\|x_{j}-v_{i}\right\|^{2}
$$

where $X=\left\{x_{1}, x_{2}, \ldots, x_{N}\right\}$ denote the set of data $\left(x_{k}\right.$ correspond to feature vector described in section 2.2). $V=$ $\left\{v_{1}, v_{2}, \ldots, v_{C}\right\}$ represents the prototypes, known as the clusters centers. $U=\left[u_{i j}\right]$ is the partition matrix which satisfies the following conditions:

$$
\begin{gathered}
u_{i j} \in[0,1] \quad \forall i, j \quad 0<\sum_{j}^{N} u_{i j}<N \quad \forall i \\
\sum_{i}^{C} u_{i j}=1 \quad \forall j
\end{gathered}
$$


$m$ is a fuzzifier which indicate the fuzziness of membership for each point. FCM algorithm is based on an iterative process by minimizing the distance between each point and the prototypes. As we can notice from Eq. 2 the FCM objective function does not incorporate any spatial constraint. This can lead to a formation of small undesirable regions. More over in presence of noise classification process is affected directly by the noise. In order to overcome this problem, the regularization term is added to constraint the Eq. 2 allowing the classification of each point to be influenced by its neighborhood. The modified objective function can be expressed as follow:

$$
\begin{aligned}
J_{M}(U, V) & =\sum_{i=1}^{C} \sum_{j=1}^{N} u_{i j}^{m}\left\|x_{j}-v_{i}\right\|^{2} \\
& +\alpha \sum_{i=1}^{C} \sum_{j=1}^{N} u_{i j}^{m} e^{-\sum_{k \in \Omega} u_{i k}^{m}}
\end{aligned}
$$

where $\Omega$ is a set of neighbors. The parameter $\alpha$ is a weight that controls the influence of the second term. The objective function (5) has two components. The first component is the same as FCM, the second is a penalty term. This component reaches a minimum when the membership value of neighbors in a particular cluster is large. The optimization of (5) with respect to $U$ will be solved by using Lagrange multiplier technique.

$$
\begin{aligned}
J_{M}(U, V) & =\sum_{i=1}^{C} \sum_{j=1}^{N} u_{i j}^{m}\left(\left\|x_{j}-v_{i}\right\|^{2}+\alpha e^{-\sum_{k \in \Omega} u_{i k}^{m}}\right) \\
& +\sum_{j=1}^{N} \lambda_{j}\left(1-\sum_{i=1}^{C} u_{i j}\right)
\end{aligned}
$$

the derivative of (6) with respect to $u_{i j}$

$$
\frac{\partial J_{M}}{\partial u_{i j}}=m u_{i j}^{m-1}\left(\left\|x_{j}-v_{i}\right\|^{2}+\alpha e^{-\sum_{k \in \Omega} u_{i k}^{m}}\right)-\lambda_{j}
$$

solving for $u_{i j}$ we have

$$
u_{i j}=\left(\frac{\lambda_{j}}{m\left(\left\|x_{j}-v_{i}\right\|^{2}+\alpha e^{\left.-\sum_{k \in \Omega} u_{i k}^{m}\right)}\right.}\right)^{\frac{1}{m-1}}
$$

solving for $\lambda_{j}$ with respect to the constraint (4) we obtain

$$
\sum_{i=1}^{C}\left(\frac{\lambda_{j}}{m\left(\left\|x_{j}-v_{i}\right\|^{2}+\alpha e^{-\sum_{k \in \Omega} u_{i k}^{m}}\right)}\right)^{\frac{1}{m-1}}=1
$$

As $\lambda_{j}$ does not depend in the term of the sum this yield

$$
\lambda_{j}^{\frac{-1}{m-1}}=\sum_{i=1}^{C}\left(m\left\|x_{j}-v_{i}\right\|^{2}+\alpha e^{-\sum_{k \in \Omega} u_{i k}^{m}}\right)^{\frac{-1}{m-1}}
$$

substituting in (8) we obtain the following update membership

$$
u_{i j}=\frac{1}{\sum_{p=1}^{C}\left(\frac{\left\|x_{j}-v_{i}\right\|^{2}+\alpha e^{-\sum_{k \in \Omega} u_{i k}^{m}}}{\left.\left\|x_{j}-v_{p}\right\|^{2}+\alpha e^{-\sum_{k \in \Omega^{u}}^{m}}\right)^{\frac{1}{m-1}}}\right.}
$$

As seen from (11), the neighboring membership values $\left(u_{p k}\right)$ influence $u_{i j}$ to follow the neighborhood behavior. For instance if a given point has a high membership value to a particular cluster and its spatial neighbors have a small membership values to this cluster, the penalty term plays the role to force the point to belong to the same cluster as its neighbors. As we can notice from (11), the weight $\alpha$ controls the importance of the regularization term. In the particular case when $\alpha=0$ the membership value, $u_{i j}$, is independent of the neighborhood membership values and it turn out the same standard FCM membership function. If $\alpha$ is too large, the segmentation result will be very smooth and the shape of regions can be affected. The value $\alpha$ should be chosen with respect to trade-off between both terms.

The prototype update equation is the same as standard FCM, since the second component of (5) does not depend on $v_{i}$. Thus we obtain the centroids update by the following equation:

$$
v_{i}=\frac{\sum_{j=1}^{N} u_{i j}^{m} x_{j}}{\sum_{j=1}^{N} u_{i j}^{m}}
$$

The Modified FCM (MFCM) algorithm can be summarized in the following steps:

Step 1 Fix the number of clusters

Initialize $u_{i j}$ by random value $\in[0,1]$

Initialize the centers by random points from data set

Step 2 Compute the distance $\left\|x_{j}-v_{i}\right\|^{2}$

Step 3 Update the partition matrix using (11)

Step 4 Update centroids using (12)

Step 5 Repeat step 2 to step 5 till convergence

The convergence of the process is considered here to be reached when the change in membership values is less than the threshold.

\section{EXPERIMENTS}

In this section we illustrate the application of the proposed algorithm on synthetic images corrupted by noise and remote sensing (RS) images. In all experiences the parameter $\alpha$ was set to $70, \mathrm{w}=0.5$ and $m$ is chosen to be 2 .

Fig. 3 (a) shows a synthetic image containing five class patterns corrupted by $20 \%$ of shotnoise. Fig. 3 (b) shows the result of applying FCM to the original image. As we can 


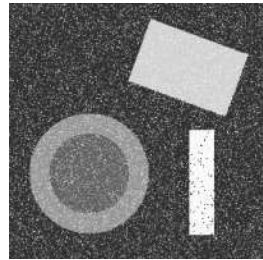

(a)

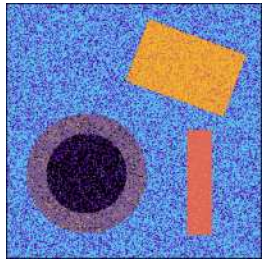

(b)

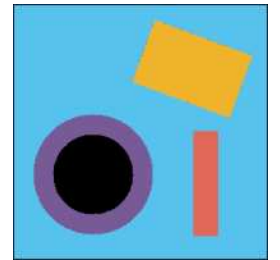

(c)
Fig. 3. Segmentation results using FCM and MFCM algorithms for synthetic images

see FCM algorithm is not able to segment correctly the original image. This example shows us the sensitivity of FCM to noise and other image artifacts. It is due to that FCM classify pixels without taking into account the spatial dependence of pixels. Application of MFCM yields the segmentation shown in Fig. 3 (c). The five classes are correctly detected.

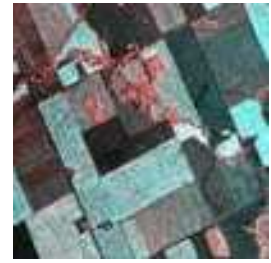

(a)

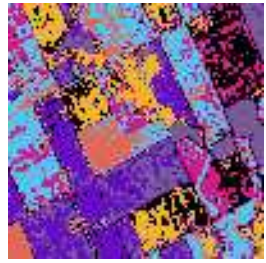

(b)

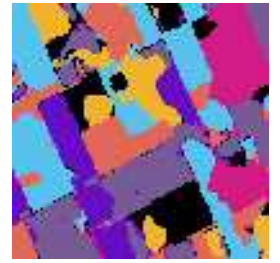

(c)
Fig. 4. Segmentation results using FCM and MFCM for RS images

Real multispectral test images were obtained from CCRS (http://www.ccrs.nrcan.gc.ca). Fig. 4 (a) represents agricultural fields. The image was created by $\mathrm{C} / \mathrm{X}$ band SAR system: in each pixel 3 bands represent the spectral characteristics. This images contain many kinds of noise and artifacts due to the sensor, atmospheric distortions.... Such conditions make it difficult to segment such images by classical methods as FCM. The segmentation results yielded by FCM and MFCM are shown respectively in Figs. 4 (b)(c). We can notice that FCM segmentation shows a lot of insignificant regions. Comparing to that, MFCM provides more accurate segmentation. The proposed method outperforms FCM and brings out a good segmentation performance: the difference with FCM remains qualitatively consistent on samples of a dozen of similar images(eg. agricultural, forest, urban,...).

\section{CONCLUSION}

In this paper we proposed a new method for segmentation. A new feature and a new FCM objective function are used to incorporate spatial information, aiming for more effec- tive segmentation. We compared our results with traditional FCM. The MFCM proves more robust than FCM on both synthetic and RS images. In future works more criteria will be considered for additional constraints such as automatic determination of the number of classes and probabilistic methods to calculate weights.

\section{REFERENCES}

[1] A. K. Jain, M. N. Musty and P. j Flynn "Data Clustering: A Review," ACM Comput Surv, Vol. 31. No 3, pp. 264-323, 1999.

[2] L. Kaufman and P. Rousseeuw "Finding Groups in Data, An Introduction to Cluster Analysis," John Wiley\&Sons, 1990.

[3] J. C. Bezdek "Pattern Recognition With Fuzzy Objective Function Algorithm," Plenum Press, New York, 1981.

[4] R. Krishnapuram and J. Keller "Fuzzy and Possibilistic Clustering Methods for Computer Vision," Neural and Fuzzy Systems, Vol. 31. No 3, pp. 133-159, 1994.

[5] Y. A. Tolias and S. M. Panas "On applying Spatial Constraints in Fuzzy Image Clustering Using a Fuzzy RuleBased System ," IEEE Signal Processing Letters, Vol. 5, pp. 245-247, 1998.

[6] D. L. Pham "Spatial Models for Fuzzy Clustering," Computer Vision and Image Understanding, Vol. 84. No 2, pp. 285-297, 2001.

[7] M. N. Ahmed, S. M. Yamany, N. Mohamed, A. A. Frag and T. Moriatry "A Modified Fuzzy C-means Algorithm for Bias Field Estimation and Segmentation of MRI Data," IEEE Trans. on Medical Imaging, Vol. 21 No 3, pp. 193-199, 2002.

[8] S. Z. Li "Markov Random Field in Computer Vision," Springer-Verlag, 1995.

[9] T. Bially "Space-filling Curves: Thier Generation and Thier Application to Bandwidth Reduction," IEEE Transaction on Information Theory, Vol 5, pp 658-664.

[10] G. Seetharaman and B. Zavidovique "Z-Trees: Adaptive Pyramid Algorithms for Segmentation," IEEE International Conference on Image Processing,Chicago, IL, October 98.

[11] N. Jhanwar, S. Chaudhuri, G. Seetharaman and B. Zavidovique "Content Based Image Retrieval using Motif Cooccurrence Matrix," Image and Vision Computing, Vol. 22, pp 1211-1220, 2004. 